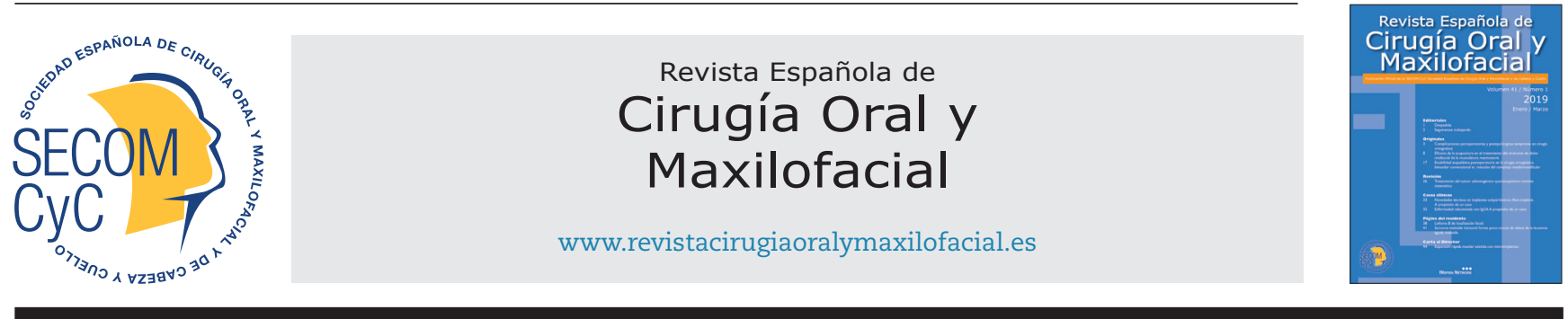

Página del residente

\title{
Sarcoma mieloide intraoral: forma poco común de debut de la leucemia aguda mieloide
}

\author{
Cristina Vázquez-Martínez, Marta Redondo-Alamillos, Ana I. Romance-García y \\ Gregorio Sánchez-Aniceto
}

Servicio de Cirugía Oral y Maxilofacial. Hospital Universitario 12 de Octubre. Madrid, España

\section{INFORMACIÓN DEL ARTÍCULO}

Historia del artículo:

Recibido el 13 de enero de 2018

Aceptado el 03 de junio de 2018

Palabras clave:

Leucemia aguda mieloide, sarcoma mieloide, neoplasia cavidad oral.

\section{Keywords:}

Acute myeloid leukemia, myeloid sarcoma, oral neoplasm.

\section{R E S U M E N}

La leucemia aguda mieloide (LAM) es una neoplasia maligna con manifestaciones sistémicas, pudiendo presentar lesiones a nivel intraoral. Es el cáncer más frecuente en la infancia y constituye la primera causa de muerte por cáncer en la edad pediátrica. El sarcoma mieloide es un tumor maligno extramedular compuesto por células precursoras mieloides inmaduras. Este tumor se asocia fundamentalmente con la leucemia mieloide aguda y síndromes mielodisplásicos y, generalmente, debuta después del diagnóstico de la enfermedad primaria. La aparición del sarcoma mieloide dentro de la cavidad oral como manifestación inicial de la LAM es extremadamente rara (3-6 \% de los casos según las series).

Se presenta el caso de una lactante de 19 meses de edad que llevaron a Urgencias por fiebre y una tumoración gingival en el cuarto cuadrante refractaria a tratamiento antibiótico. Se solicitó un estudio ecográfico para valorar la existencia de un absceso, donde se identificó una lesión que, tras realizar una biopsia para estudio anatomopatológico, resultó ser un sarcoma mieloide. La paciente fue diagnosticada de LAM y se inició tratamiento quimioterápico. Tras tres ciclos, actualmente la paciente se encuentra libre de enfermedad.

\section{A B S T R A C T}

Acute myeloid leukemia (AML) is a malignant neoplasm with systemic manifestations, which may present intraoral lesions. It is the most common cancer in childhood and the leading cause of death from cancer in pediatric population. Myeloid sarcoma is a malignant extramedullary tumor composed of immature myeloid precursor cells. This tumor is mainly associated with AML and myelodysplastic syndromes and, generally, shows up after the diagnosis of the primary disease. The appearance of myeloid sarcoma within the oral cavity as an initial manifestation of AMK is extremely rare (3-6\% of cases according to the series).

\footnotetext{
*Autor para correspondencia

Correo electrónico: cvmartinez@salud.madrid.org (Cristina Vázquez Martínez).

https://doi.org/10.20986/recom.2019.1030/2019
}

1130-0558/@ 2019 SECOM. Publicado por Inspira Network. Este es un artículo Open Access bajo la licencia CC BY-NC-ND (http:// creativecommons.org/licenses/by-nc-nd/4.0/). 
We present a case of a 19-month infant who was taken to the Emergency Department due to fever and a gingival lesion that did not respond to antibiotic treatment. An ultrasound study was requested to assess the presence of an abscess. However, a lesion was identified and after performing a biopsy, pathologist found it was a myeloid sarcoma.

The patient was diagnosed with AML and chemotherapy treatment was started. After three cycles of treatment, the patient is currently free of disease.

\section{Presentación del caso}

Se presenta el caso de una niña de 19 meses de edad que acudió en enero de 2016 al Servicio de Urgencias del Hospital Universitario 12 de Octubre por fiebre, disminución del apetito e irritabilidad de 4 días de evolución, sin presentar mejoría tras un ciclo de antibioterapia.

En la exploración física se objetivó una lesión inflamatoria en el cuerpo mandibular derecho y movilidad de la pieza 85. Ante estos hallazgos se solicita una ecografía de partes blandas en la que se describe una masa hipoecogénica de $13 \mathrm{~mm}$ en el cuerpo mandibular derecho, en probable relación con un germen dentario (Figura 1).

Ante la sospecha inicial de un quiste de erupción dental sobreinfectado, se procedió a la exodoncia de la pieza 85 y legrado de un tejido friable que se envió para estudio anatomopatológico (AP) y microbiológico. Además se solicitó una analítica sanguínea, destacando 165.000 leucocitos con un $80 \%$ de blastos en el frotis sanguíneo, 95.000 plaquetas, hemoglobina 11,7 g/dl, PCR 10 g/dl y LDH 2478 U/l. En la ecografía abdominal destacó una hepatomegalia inespecífica compatible con hepatopatía por leucosis.

El resultado AP fue de sarcoma mieloide (SM) (Figura 2) y en la microbiología se halló flora mixta. El estudio inmunohistoquímico de la pieza reveló células neoplásicas CD43+ mieloperoxidasa negativas. El inmunofenotipo de médula ósea mostró presencia de células inmaduras de estirpe monocítica: CD64+, CD36+, HLADR+, CD33+, CD33+, CD11b+, CD15+, CD14+, con

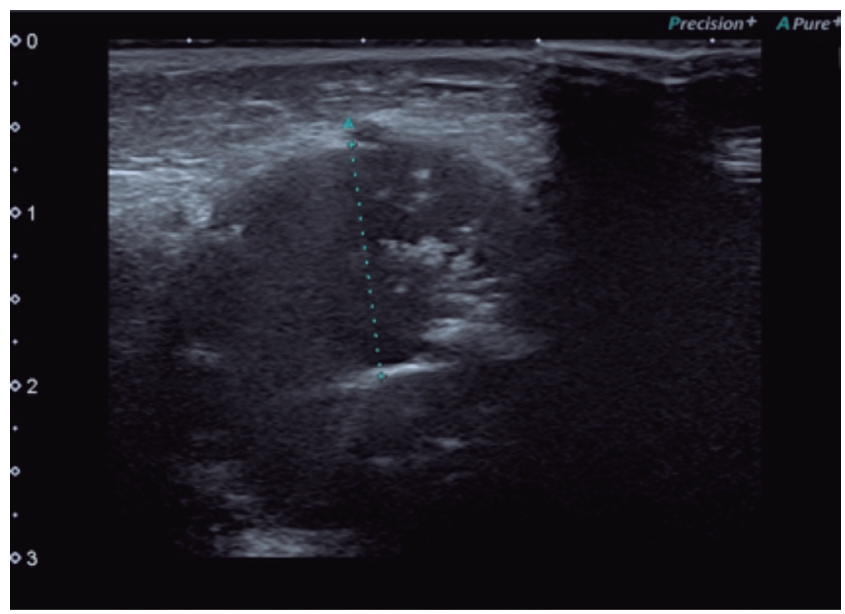

Figura 1. Imagen ecográfica: lesión intraoral hipoecogénica, sugestiva de absceso. negatividad para CD13 y expresión de CD56. El aspirado mostró una MO hipercelular, con una infiltración por blastos del $74 \%$ (Figura 3).

Tras ser diagnosticada de LAM-M5b según la clasificación Francesa-Americana-Británica, se inició tratamiento quimioterápico. En total, la paciente recibió tres ciclos, encontrándose actualmente en remisión completa.

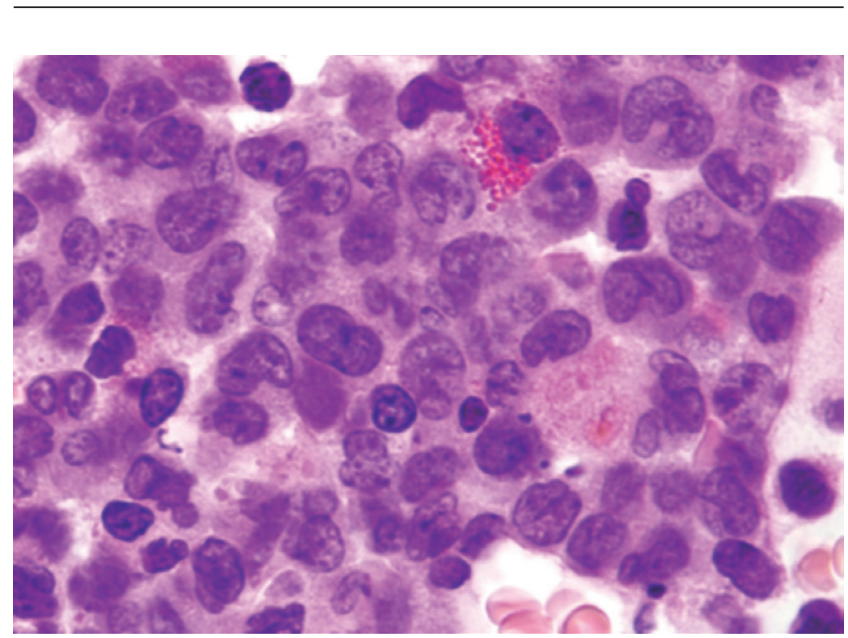

Figura 2. Proliferación difusa de células intermedias con escaso citopasma eosinófilo y núcleos muy irregulares con profundas hendiduras. Células CD47+y lisozima. H-E 100x.

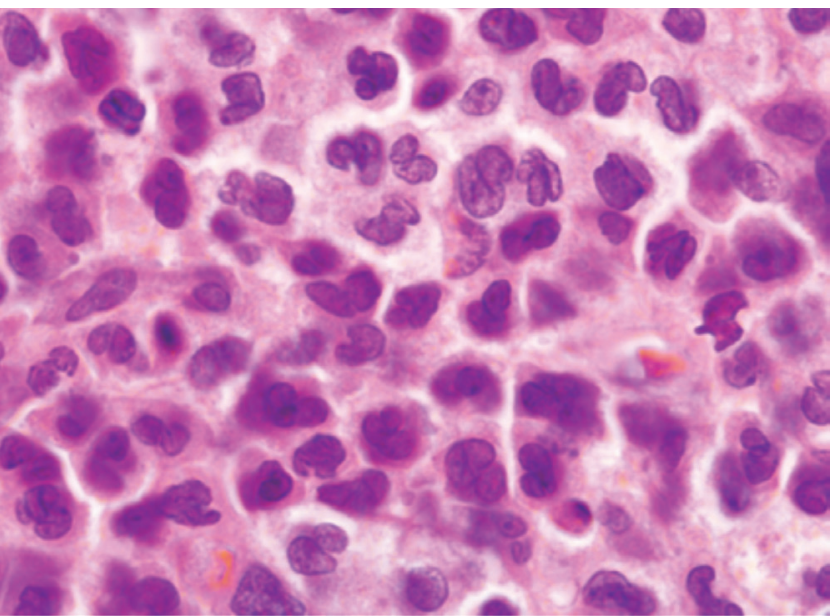

Figura 3. Estudio MO: Infiltración difusa con células similares al tumor primitivo. H-E 100x. 


\section{Discusión}

La leucemia aguda mieloide es una neoplasia mieloide derivada de la proliferación clonal de precursores hematopoyéti$\cos ^{1}$. Es la forma más común de cáncer por debajo de los 24 años y la primera causa de muerte por cáncer en la infancia ${ }^{2}$.

Cursa con manifestaciones generales y otras secundarias a la enfermedad, como infecciones recurrentes, dolor óseo o abdominal, sangrado y púrpuras hemorrágicas ${ }^{3}$. En cuanto a las manifestaciones intraorales, suele presentarse como una masa única e indolora ${ }^{3,4}$ en la encía, con tendencia al sangrado espontáneo. También son frecuentes las úlceras orales y las infecciones intraorales por hongos, bacterias o virus (gingivoestomatitis herpética) debido a la inmunosupresión ${ }^{2}$. El SM es una forma muy rara de manifestación de la leucemia a nivel intraoral, habiéndose reportado muy pocos casos hasta la fecha ${ }^{5}$.

A lo largo de la historia, el SM ha recibido varios nombres: cloroma, sarcoma granulocítico y tumor mieloide extramedu$\operatorname{lar}^{3,4,6}$. Fue descrito por primera vez por Burns en 1811, quien le concedió el nombre de cloroma, palabra de origen griego que significa "verde", debido a los altos niveles de la enzima mieloperoxidasa que es la que produce el tono verdoso de este tipo de tumores ${ }^{3,4}$. En 1966, Rappaport descubrió que dicha coloración no se encuentra siempre presente, motivo por el que lo denominó sarcoma granulocítico ${ }^{4}$.

El SM puede aparecer a cualquier edad, aunque en dos tercios de los casos se manifiestan antes de los cuatro años ${ }^{6}$, asociándose a la LAM en un 3-4,7 \% de los $\operatorname{casos}^{1,3,4}$.

La forma de aparición más común del SM intraoral es en la encía vestibular ${ }^{4}$. La manifestación intraósea es extremadamente $\operatorname{rara}^{4,6} \mathrm{y}$, si aparece, suele ser como una masa única e indolora, siendo la localización multifocal infrecuente 4 .

Radiológicamente aparece como una radiolucencia que produce destrucción de la cortical ósea ${ }^{4}$.

El diagnóstico diferencial se establece con carcinomas, un granuloma piogénico, periodontitis, abscesos periapicales ${ }^{3,4}$, linfomas ${ }^{7}$, metástasis o con el sarcoma de Ewing ${ }^{3}$.

El diagnóstico se completa con un estudio AP, inmunohistoquímico y por citometría de flujo ${ }^{3,4}$. El estudio inmunohistoquímico muestra positividad para los anticuerpos contra lisozima, mieloperoxidasa y cloroacetato esterasa ${ }^{3,4,6}$.
El tratamiento se basa en QT asociada o no a radioterapia.

Aunque no hay estudios en términos de supervivencia, se sabe que el pronóstico empeora con la edad, el retraso en el diagnóstico y si aparece infiltración gingival ${ }^{3}$.

\section{Agradecimientos}

A la Sección de Cabeza y Cuello del Servicio de Anatomía Patológica del Hospital Universitario 12 de Octubre.

\section{Conflicto de intereses}

Los autores declaran que no existe conflicto de intereses.

\section{B I B L I O G R A F Í A}

1. Babu SPKK, Kashyap V, Sivaranjani P, Agila S. An undiagnosed case of acute myeloid leukemia. J Indian Soc Periodontol. 2014;18(1):95-7. DOI: 10.4103/0972-124X.128257.

2. Guan G, Firth N. Oral manifestations as an early clinical sign of acute myeloid leukaemia: a case report. Aust Dent J. 2015;60(1):123-7. DOI: 10.1111/adj.12272.

3. Papamanthos MK, Kolokotronis AE, Skulakis HE, Fericean A-MA, Zorba MT, Matiakis AT. Acute myeloid leukaemia diagnosed by intra-oral myeloid sarcoma. A case report. Head Neck Pathol. 2010;4(2):132-5. DOI: 10.1007/s12105010-0163-9.

4. Kurdoğlu B, Oztemel A, Banş E, Sengüven B. Primary oral myeloid sarcoma: Report of a case. J Oral Maxillofac Pathol JOMFP. 2013;17(3):413-6. DOI: 10.4103/0973-029X.125209.

5. Yap M, Hewson I, McLean C, Ciciulla J. Oral myeloid sarcoma: two case reports. Aust Dent J. 2014;59(4):511-5. DOI: 10.1111/ adj.12220.

6. Jordan RCK, Glenn L, Treseler PA, Regezi JA. Granulocytic sarcoma: case report with an unusual presentation and review of the literature. J Oral Maxillofac Surg Off J Am Assoc Oral Maxillofac Surg. 2002;60(10):1206-11.

7. Ahmad J, Zafar L, Hussain G, Kausar S. Myeloid sarcoma in a child with acute myeloblastic leukaemia. J Coll Physicians Surg--Pak JCPSP. 2011;21(6):369-70. DOI: 07.2011/JCPSP.369370 